\title{
Spontaneous regression of retinoblastoma in identical twins
}

\author{
CLIVE MIGDAL \\ From the Ocular Oncology Clinic, St Bartholomew's Hospital, London EC1A 7BE
}

SUMMARY Spontaneous regression of retinoblastoma is described in identical twin boys. In the one bilaterally affected child 2 tumours regressed spontaneously, while a third lesion, which developed at a later stage, required treatment in the form of a cobalt plaque. The second child showed spontaneous regression of the single tumour in his only affected eye. The pathogenesis of spontaneous regression is discussed and a possible common aetiology in these 2 cases postulated.

Spontaneous regression of retinoblastoma is a term used by eye pathologists when calcified nests of tumour cells are found in an enucleated, often phthisical, eye, and by clinical ophthalmologists when fundal examination of a patient with retinoblastoma or relative has revealed, usually, an elevated calcified white mass, surrounded by areas of chorioretinal atrophy (no treatment ever having been given).

Retinoblastoma is unusual in the high incidence of spontaneous regression encountered $(1 \cdot 8 \%) .{ }^{1-6}$ Several cases of bilateral retinoblastoma showed inert tumour in one eye with viable retinoblastoma in the fellow eye, while the spontaneous regression occurred in both eyes in only 12 of 47 cases reported. ${ }^{7 \rightarrow 9}$ Boniuk and Zimmerman ${ }^{2}$ reported a pair of identical twins with unilateral retinoblastoma in whom the tumour spontaneously regressed in one child whereas an enucleation was required in the second child for a viable tumour.

This paper reports clinical spontaneous regression of retinoblastoma in a pair of identical twin boys in whom there was a positive family history of retinoblastoma.

\section{Case reports}

\section{CASE 1}

A Caucasian boy, one of a pair of identical twins born on 15 March 1973, was referred to an ophthalmologist at the age of 4 years 3 months after a health visitor had suspected reduced vision in the left eye. The children

Correspondence to Clive Migdal, FRCS, Eye Department, St Bartholomew's Hospital, West Smithfield, London EC1A 7BE. had been born 5 weeks prematurely, but milestones were normal. The only illness suffered was a bout of 'croup' at the age of $2 \frac{1}{2}$ years.

A positive family history of retinoblastoma was obtained, the father having had his right eye enucleated at the age of 3 years with the diagnosis proved histologically.

Examination under anaesthesia in July 1977 revealed spontaneous regression of tumours in both eyes. A small, inert tumour $3 \mathrm{~mm}$ in diameter was in

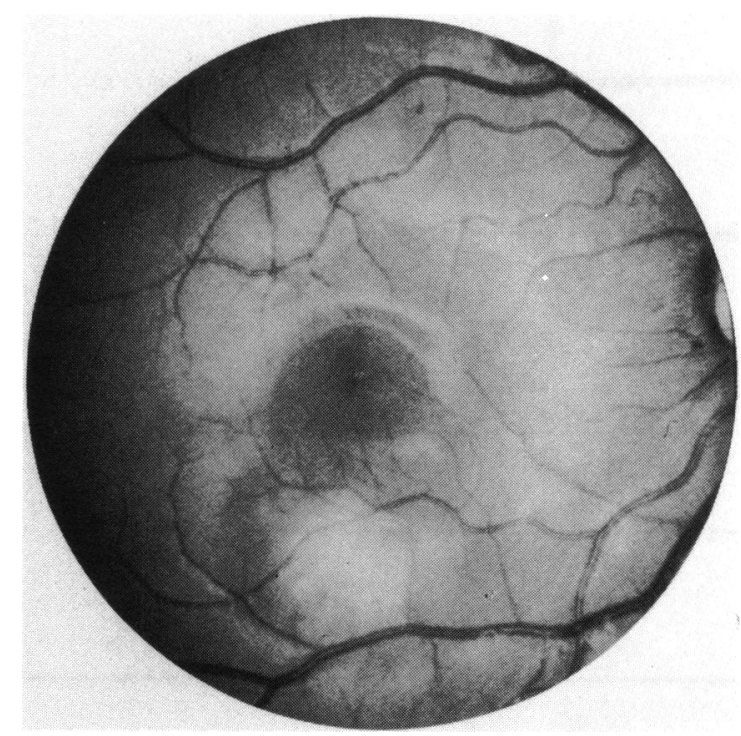

Fig. 1 Case 1. Right eye. Inert retinoblastoma after spontaneous regression. 


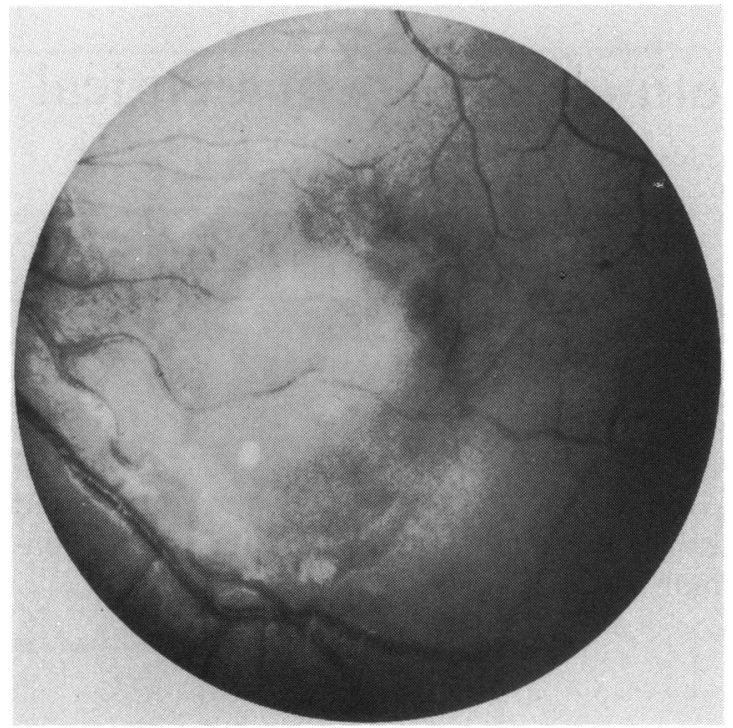

Fig. 2 Case 1. Left eye. Paramacular lesion after spontaneous regression.

the inferotemporal quadrant of the right eye below the macula (Fig. 1) while a paramacular lesion $5 \mathrm{~mm}$ in diameter (Fig. 2), with patches of calcification, was noted in the left eye. No evidence of past iritis was present.

On examination 6 weeks later a new, active tumour

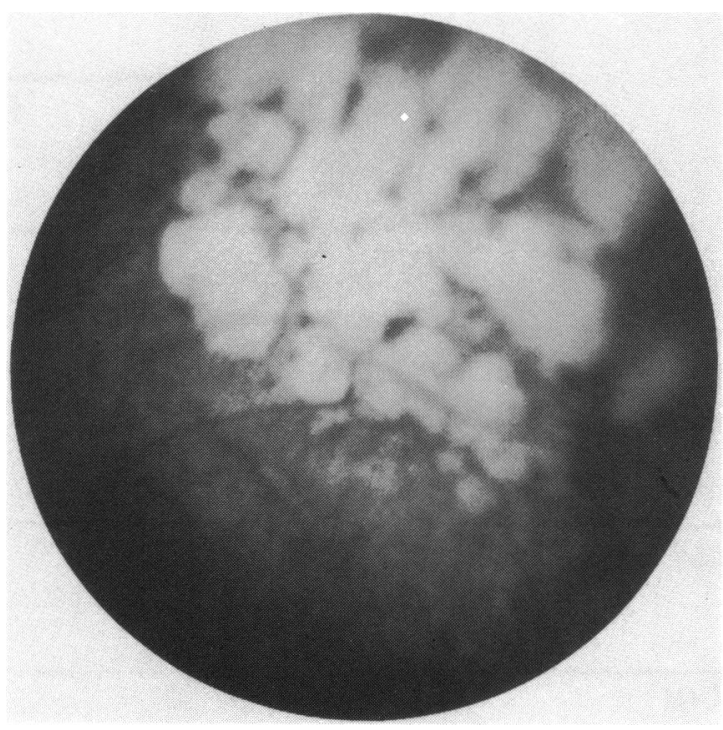

Fig. 3 Case 1. Right eye. Inert retinoblastoma after cobalt plaque irradiation.

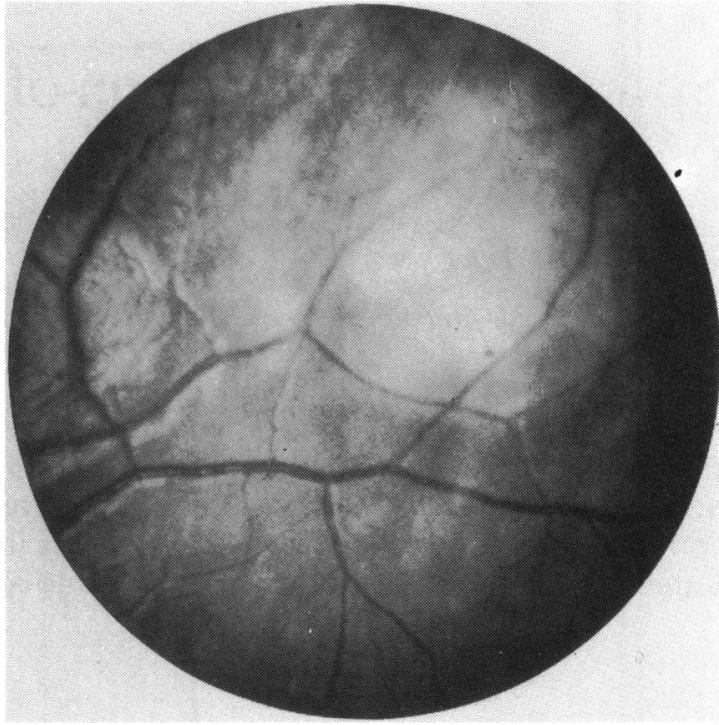

Fig. 4 Case 2. Left eye. Spontaneously regressed retinoblastoma.

was noted in the upper nasal periphery of the right eye. This required focal radiation in the form of a D-shaped $10 \mathrm{~mm}$ cobalt plaque. The 2 original tumours remained unchanged.

Subsequent follow-up showed a good response to treatment. The lesion has remained inert until the present time (Fig. 3), as have the 2 other foci initially discovered.

The vision in the right eye is $6 / 9$ unaided, and that in the left eye $6 / 36$.

CASE 2

This boy was seen by an ophthalmologist after the diagnosis of bilateral retinoblastoma had been made in his identical twin brother (case 1). He had no visual symptoms and had no medical illnesses apart from mumps at the age of 3 years 9 months.

On examination under anaesthesia in July 1977 a spontaneously regressed tumour $5 \mathrm{~mm}$ in diameter was noted above the left macula. The right eye, however, was normal. At subsequent examinations the tumour has remained inert (Fig. 4) and has not required any treatment. No new lesions have developed.

CYTOGENETIC INVESTIGATIONS

The twins were typed for 16 different blood-group factors and they were identical in every respect. The frequency of polymorphic types and likeness of sex indicate a possibility for dizygotic twinning of only 0.02 (i.e., $98 \%$ chance that these twins are monozygotic). This probability is improved still further by 
the fact that their dermatoglyphic features were so very similar, both in pattern type and size, hand for hand. In summary, therefore, there is a $99 \%$ certainty of identical twinning. Their karyotypes were normal, without evidence of a $13 q$ deletion.

\section{IMMUNOLOGICAL STUDY}

Lymphocyte transformation tests (March 1982). Allogeneic tumour cells obtained from enucleated eyes were suspended in $20 \mathrm{ml}$ of $3 \mathrm{M} \mathrm{KCl}$ ( $\mathrm{pH} 7.4$ ) and left at $4^{\circ} \mathrm{C}$ for 24 hours with frequent shaking. The suspension was centrifuged at $40000 \mathrm{~g}$ for 60 minutes and the supernate was dialysed for 16 hours against phosphate-buffered saline. The material was concentrated with lyphogel and sterilised by passing through a Millipore filter $(0.22 \mu \mathrm{m})$.

The protein concentration was estimated by a modified Lowry method. Blood from the 2 cases was collected in preservative-free heparin ( 20 units per $\mathrm{ml}$ ) and lymphocytes were separated by a density gradient technique using Ficoll-Hypaque preparation. Lymphocytes were cultured in medium 199 enriched with freshly prepared glutamine (2 $\mathrm{mmol} / \mathrm{ml})$ and the patients' own serum (1:10).

The test was carried out in quadruplicate in a microtitre plate in a gaseous phase of $5 \%$ carbon dioxide. Various concentrations of tumour extracts were added to the lymphocyte culture 24 hours after the initial incubation; $0.4 \mu \mathrm{Ci}$ of tritiated thymidine was added to each control and to experimental wells on the 6th day of the culture and incubated for a further period of 16 hours.

The lymphocytes were then harvested with a semiautomated harvester. The filter paper discs were transferred to scintillation vials and dried at $37^{\circ} \mathrm{C}$ for 3 hours; $10 \mathrm{ml}$ of toluene-based scintillation fluid was added to each vial, and the radioactivity was measured in a Packard tricarb liquid-scintillation counter. The result was expressed as the lymphoproliferative - that is, transformation-index.

Case 1. The transformation index with allogeneic retinoblastoma was less than 2 , which suggests that the patient was only weakly sensitised to tumour antigens.

Case 2. The transformation index with allogeneic retinoblastoma extract was 6 , which suggests autosensitisation.

\section{Discussion}

The diagnosis of spontaneous regression of retinoblastoma in the above cases has been made on the basis of the characteristic clinical findings in the presence of a family history of retinoblastoma. The ophthalmoscopic appearance in spontaneous regression characteristically consists of one or more

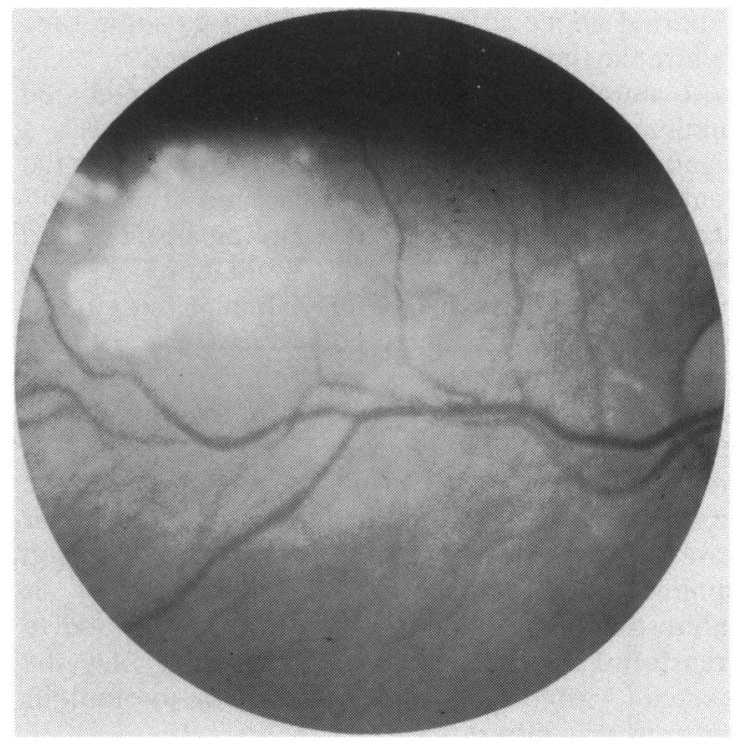

Fig. 5 Regression pattern of retinoblastoma after external beam radiotherapy with the cobalt beam (3500 rads).

areas of chorioretinal atrophy in which elevated, irregular collections of chalk-white calcifications can be seen, often having the appearance of cottage cheese. Abnormal blood vessels are often found in the region of the tumour. This picture is similar to that following radiotherapy for retinoblastoma (Fig. 5).

The pathogenesis of spontaneous regression of retinoblastoma has not yet been fully established, but various hypotheses have been suggested to explain its occurrence, in particular tumour ischaemia and the host's immunological response. In the general sense growth of tumour cells is limited by vascular stasis and thrombosis resulting in capillary blockage in the neoplasm, relative to the proliferation and mass of the tumour itself. Areas of ischaemia may be accounted for by scanty stromal vascularisation, which in turn may be secondary to a lack of tumour angiogenesis factor or the inhibition of such a factor, either by some environmental factor or by an immune response. ${ }^{10}$ Ischaemia alone, either accidental or from the tumour outgrowing its blood supply, is an unlikely explanation for the regression in these 2 cases of relatively small tumours.

It was suggested " that an acute febrile illness can trigger off a regression in the report of a case in which regression took place during scarlet fever at the age of 4 years. The fellow eye had been removed previously owing to retinoblastoma. Spontaneous regressions associated with acute febrile illnesses and other nonspecific Freund's-adjuvant-type stimuli have been described in other malignancies, such as melanomas and carcinoma of the colon. ${ }^{12}$ Both the 2 cases 
reported above suffered febrile illnesses some time before the tumours were initially discovered.

It must be presumed that the cell-mediated immune response to tumour antigens plays a significant part in the defence of the host against the tumour. In-vitro studies by Char and coworkers ${ }^{13}$ showed high levels of cytotoxicity against labelled retinoblastoma cells in the evaluation of cellmediated immunity to a retinoblastoma tissue culture line. In addition a cutaneous delayed hypersensitivity response was also elicited from the skin testing of patients with retinoblastoma with crude membrane extracts of retinoblastoma tissue culture cells. ${ }^{14} \mathrm{~A}$ lowered host immune response may therefore be postulated in case 1 of this report and in other similar cases $^{3}$ in which some tumours regressed spontaneously, while others advanced. It is interesting that the results of the lymphocyte transformation test in case 1 suggested that the patient was only weakly sensitised to tumour antigens, in contrast to case 2 .

Some local factor may be responsible in addition to the above. Calcium is an inhibitor of tumour growth, and it has been suggested that the excessive calcification often found in tumours showing spontaneous regression might lead to a toxic concentration of calcium and self-destruction of the tumour. However, it has never been proved that spontaneous regression is an effect of the calcification (which is also found in inert tumours treated by radiotherapy). The presence of the calcium deposits may, however, predispose the tumour cells to one of the other processes discussed above, which may in turn lead to spontaneous regression.

In conclusion, it is possible to postulate in the 2 cases reported above that some systemic factor, immunological or other and common to both children, allowed the spontaneous destruction of the tumours initially present. However, in one twin a subsequent tumour was not affected by this process and required treatment. Whether this would also have regressed spontaneously if the natural course of progression over a longer period was permitted is uncertain.

I am indebted to Michael Bedford, FRCS, for his permission to publish these cases, to Drs Rahi and Butler for their help with the immunological and cytogenetic investigations respectively, and to Sarah Dew for secretarial assistance.

\section{References}

1 Steward JK, Smith JLS, Arnold EL. Spontaneous regression of retinoblastoma. Br J Ophthalmol 1956; 40: 449-61.

2 Boniuk M. Zimmerman LE. Spontaneous regression of retinoblastoma. Int Ophthalmol Clin 1962; 2: 525-42.

3 Karsgaard AT. Spontaneous regression of retinoblastoma. Can J Ophthalmol 1971; 6: 218-22.

4 Morris WE, La Piana FG. Spontaneous regression of bilateral multifocal retinoblastoma with preservation of normal vision. Ann Ophthalmol 1974; 6: 1192-4.

5 Lindley J, Smith S. Histology and spontaneous regression of retinoblastoma. Trans Ophthalmol Soc UK 1974; 94: 953-67.

6 Nehen JH. Spontaneous regression of retinoblastoma. Acta Ophthalmol (Kbh) 1975; 53: 647-51.

7 Boniuk M. Girard LJ. Spontaneous regression of bilateral retinoblastoma. Trans Am Acad Ophthalmol Otolaryngol 1969; 73: 194-8.

8 Khodadoust AA. Roozitalab HM, et al. Spontaneous regression of retinoblastoma. Surv Ophthalmol 1977; 21: 467-8.

9 Brodwall J. Spontaneous regression of a retinoblastoma. Acta Ophthalmol (Kbh) 1981; 59: 430-4.

10 Sang DN, Albert DM. Recent advances in the study of retinoblastoma. In: Jakobiec F, ed. Ocular and adnexal tumours. Birmingham, Alabama: Aesculapius, 1978: 285-329.

11 Stallard HB. Glioma retinae treated by radon seeds. $\mathrm{Br}$ Med J 1936; ii: $962-4$

12 Cole WH. Spontaneous regression of cancer: the metabolic triumph of the host? Ann NY Acad Sci 1974; 230: 111-41.

13 Char DH, Elsworth RM, Rabson A, et al. Cell-mediated immunity to a retinoblastoma tissue culture line in patients with retinoblastoma. Am J Ophthalmol 1974; 78: 5-11.

14 Char DH, Herberman RB. Cutaneous delayed hypersensitivity responses of patients with retinoblastoma to standard recall antigens and crude membrane extracts of retinoblastoma tissue culture cells. Am J Ophthalmol 1974; 78: 40-4. 